\title{
RELATOS DE PESQUISAS \\ SISTEMAS DE INFORMAÇÕES PARA GERENCIAMENTO DE RISCOS CORPORATIVOS EM CONTROLADORIA: UM ESTUDO BIBLIOMÉTRICO NA BASE DE DADOS SCOPUS
}

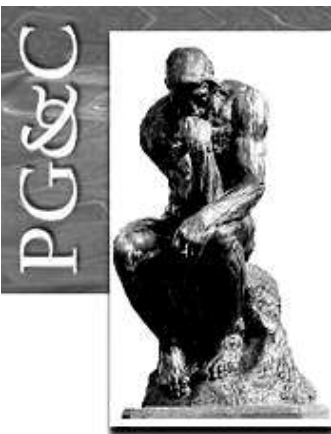

\author{
Eduardo Vieira do Prado \\ Doutorando em Administração Universidade Metodista de Piracicaba, \\ Brasil. Professor da Faculdade Santa Lúcia Mogi Mirim, Brasil. \\ E-mail: eduardo.prado.ad@gmail.com
}

Valéria Rueda Elias Spers

Doutora em Ciências Sociais pela Pontifícia Universidade Católica de São Paulo, Brasil. Professora da Universidade Metodista de Piracicaba, Brasil.

E-mail: vrueda@unimep.br

Clovis Luis Padoveze

Doutor em Controladoria e Contabilidade pela Universidade de São Paulo. Professor da Universidade Metodista de Piracicaba, Brasil.

E-mail: cpadoveze@yahoo.com.br

André Luis Bertassi

Doutor em Administração pela Universidade Metodista de Piracicaba, Brasil. Professor da Universidade Federal de São João del Rei, Brasil.

E-mail: bertassi@ufsj.edu.br

Luiz Gustavo Camarano Nazareth

Doutorando em Administração pela Universidade Metodista de Piracicaba, Brasil. Professor da Universidade Federal de São João del Rei, Brasil.

E-mail: luizgustavo@ufsj.edu.br

\begin{abstract}
Resumo
Nos últimos anos, as organizações vêm sendo demandadas a implantar boas práticas de Governança Corporativa por meio de princípios como: transparência, prestação de contas, equidade e responsabilidade corporativa. Dentre as boas práticas de Governança Corporativa destaca-se o Gerenciamento de Riscos Corporativos (GR), as quais visam planejar e desenvolver estratégias para minimização das exposições que cercam as organizações e consequentemente uma melhor preparação para aproveitamento das oportunidades existentes. No entanto, O GR somente pode ser aplicado se os tomadores de decisão tiverem informações no tempo e forma adequadas. Neste contexto, a área de Controladoria possui papel fundamental, visto que possui uma posição estratégica nas organizações na estruturação de sistemas de informações para planejamento e controle. Desta forma, este trabalho teve como objetivo geral mapear as pesquisas sobre o papel da Controladoria na estruturação de sistemas de informação para GR. Como procedimento metodológico utilizou-se: Pesquisa bibliográfica sobre os temas Controladoria e GR; Pesquisa exploratória para levantamento bibliométrico na base de dados Scopus com base na palavra-chave risco em trabalhos divulgados no Brasil e EUA de 2010 a 2015; Análise dos dados por meio do software VOSviewer. Como resultado, dentre as lacunas observadas, destaca-se a inexistência de trabalhos que abordem sobre o papel da Controladoria na estruturação de sistemas de informação para GR, bem como a abordagem de GR nas perspectivas de pessoas, processos e tecnologia.
\end{abstract}

Palavras chaves: Gerenciamento de Riscos. Controladoria. Sistemas de Informação. Bibliometria.

Perspectivas em Gestão \& Conhecimento, João Pessoa, v. 7, n. 1, p. 191-211, jan./jun. 2017. DOI: http://dx.doi.org/10.21714/2236-417X2017v7n1p191

http://periodicos.ufpb.br/ojs2/index.php/pgc. ISSN: 2236-417X. Publicação sob Licença (cc)) EY-NC-ND 


\title{
INFORMATION SYSTEMS FOR ENTERPRISE RISK MANAGEMENT IN CONTROLLING: A BIBLIOMETRIC STUDY BY THE SCOPUS DATABASE
}

\begin{abstract}
In recent years, organizations have been demanded to implement good corporate governance practices through principles such as transparency, accountability, fairness and corporate responsibility. Amongst the best practices of Corporate Governance stands out the Corporate Risk Management (ERM), which aims to plan and develop strategies to minimize exposures surrounding organizations and consequently make them better prepared to take advantage of existing opportunities. However, the ERM can only be applied if the decision makers have information on time and in an appropriate manner. In this context, the Controlling area has key role, as it has a strategic position in international organizations in structuring information systems for planning and control. Thus, this study aimed to map the research on the role of the Controlling in structuring information systems to ERM. As methodological procedure was applied: bibliographic search on the Controlling and ERM issues; Exploratory research through bibliometric survey in the Scopus database based on keyword risk in works published in Brazil and the USA from 2010 to 2015; Data analysis through VOSviewer software. As a result, among the observed gaps, there is a lack of studies that address the role of the Controlling in structuring information systems to ERM and the ERM approach involving the perspectives of people, processes and technology.
\end{abstract}

Key words: Enterprise Risk Management. Controlling. Information System. Bibliometrics.

\section{INTRODUÇÃO}

Frente a um contexto de maior complexidade, competitividade e aumento crescente dos riscos, os empreendedores vêm sendo desafiados a implantar novos mecanismos de gestão visando à necessidade de maior transparência e gerenciamento de fatos adversos à estratégia das organizações (PRADO, 2014).

Esta prática, que no passado focava na área de finanças na comparação risco versus retorno, passou por revisões constantes, visto que as organizações estão expostas a outros riscos cujas origens não são estritamente financeiras e devem merecer uma gestão igualmente importante (PADOVEZE, 2010).

Um exemplo da complexidade deste processo de gestão são as empresas que atuam em ambiente global, as quais enfrentam uma variedade adicional de problemas que dificultam a tarefa dos empreendedores. Seis grandes fatores distinguem a administração de uma empresa nacional, conhecidas também como empresas domésticas, de uma empresa que atual no mercado internacional: a) Diferentes denominações monetárias (moedas); b) Ramificações econômicas e legais; c) Diferenças de língua; d) Diferenças culturais; e) Papel do governo (menor ou maior participação na economia); ou f) Risco político. Cada país em que a empresa opera tem suas instituições políticas e econômicas, e as diferenças institucionais entre os países podem provocar problemas significativos quando a empresa tenta coordenar e controlar as operações mundiais de suas subsidiárias (WESTON; BRIGHAM, 2004). As complexidades jurídicas e econômicas presentes nesse ambiente são significativamente diferentes das que enfrentaria uma empresa doméstica (GITMAN, 2010).

Por outro lado, o conhecimento vem sendo reconhecido como um recurso chave para o sucesso em modernas organizações. Como resultado, uma questão sobre o tema é como encontrar a melhor forma para criar, transferir e utilizar o conhecimento como uma importante preocupação para a pesquisa e prática em Administração. Iniciativas técnicas visam prover tecnologias e sistemas de informação que provê uma plataforma para suporte do conhecimento. Esta plataforma enfatiza a importância de fatores sociais e técnicos nos processos do conhecimento (HANDZIC, 2011).

Perspectivas em Gestão \& Conhecimento, João Pessoa, v. 7, n. 1, p. 191-211, jan./jun. 2017. 
Baseado neste complexo cenário, este trabalho tem como problemática: Quais as pesquisas que vem sendo realizadas no que tange ao papel da Controladoria na estruturação de sistemas de informação para o Gerenciamento de Riscos Corporativos (GR)?

Portanto, o objetivo geral da pesquisa é mapear as pesquisas sobre o papel da Controladoria na estruturação de sistemas de informação para GR, visando aumentar o conhecimento sobre estes fatores adversos nas organizações.

Como objetivos específicos destacam-se: 1) Revisão bibliográfica sobre os temas Teoria de Sistemas, Controladoria e GR; 2) Levantamento bibliométrico da base de dados Scopus sobre o tema Risco.

Desta forma, os procedimentos metodológicos, foram à pesquisa bibliográfica e exploratória com posterior análise de dados pelo software VOSViewer.

Como justificativa deste estudo, a utilização das práticas de GR vem sendo estimulada devido ao nível crescente das boas práticas de governança corporativa das organizações demandado por órgãos reguladores e principalmente dos investidores. No entanto, apesar de ser um assunto explorado de forma crescente por profissionais da área de Administração e Finanças, um fator de destaque é a escassez de pesquisas científicas sobre estruturas de GR.

$\mathrm{Na}$ base do Anpad, por exemplo, foram identificados seis trabalhos que mencionam Gerenciamento ou Gestão de Riscos no título do trabalho de 2006 a 2014, porém nenhum deles objetivou discutir ou propor uma estrutura para GR, nem mesmo a sistemas de informações para subsidiar este processo. Este aspecto é reforçado por Jordan et al (2013) que dada à importância do tema, a escassez de estudos empíricos sobre riscos é surpreendente.

Adicionalmente, os seguintes fatos reforçaram a importância do GR:

- Nível crescente das boas práticas de governança corporativa das organizações, principalmente as que possuem ações ofertadas na BM\&F BOVESPA;

- Maior exigência dos órgãos reguladores e autorreguladores e principalmente dos investidores.

- Aumento de competitividade neste ambiente globalizado, que leva as empresas a pensarem de forma contínua em boas práticas de gestão.

O trabalho está organizado em quatro partes, além da introdução. O referencial teórico aborda a Teoria dos Sistemas, GR e Controladoria. Em seguida são apresentados os procedimentos metodológicos utilizados neste estudo. Por fim, apresentam-se os resultados, as análises realizadas na base de dados SCOPUS e as considerações finais sobre principais resultados obtidos em resposta ao problema de pesquisa. Na ultima parte ainda são apresentadas as limitações do estudo e as sugestões de futuras pesquisas.

\section{REFERENCIAL TEÓRICO}

A revisão de literatura foi estruturada de forma a subsidiar conceitos relevantes sobre Teoria de Sistemas, GR e Controladoria. Entende-se que a teoria de sistemas é a que suporta os estudos sobre GR e Controladoria.

\subsection{Teoria de Sistemas}

A teoria geral dos sistemas surgiu e foi popularizada após o fim da Segunda Guerra Mundial, em 1950, a partir dos trabalhos de Ludwig von Bertanlanffy, divulgando o conceito de sistemas abertos em várias disciplinas. A Segunda Guerra Mundial mostrou a todos como os países eram mutuamente dependentes e como estes, na verdade, constituíam partes

Perspectivas em Gestão \& Conhecimento, João Pessoa, v. 7, n. 1, p. 191-211, jan./jun. 2017. 
diferenciadas de um sistema global, no qual modificações de uma parte do sistema refletiamse nas outras partes que compunham a totalidade (integradas por políticas econômicas, mundiais, regras, leis, instituições, pelo comércio e por fenômenos sociais de influência mútua) (MOTTA; VASCONCELOS, 2002).

Para Motta e Vasconcelos (2002), não se pode confundir a teoria geral dos sistemas com a perspectiva funcionalista de Talcott Parsons. No entanto, apesar das importantes diferenças, no entanto, sem dúvida a perspectiva funcionalista influenciou a teoria geral dos sistemas.

Parsons (1966) define que o sistema social é constituído pela interação dos indivíduos humanos, no qual cada membro corresponde a um ator (com objetivos, ideias, atitudes entre outros) e objeto de orientação, tanto para si mesmo como para outros atores. Desta forma, o sistema de interação é um aspecto analítico que pode ser abstraído dos processos totais de ação de seus participantes. Ao mesmo tempo, tais indivíduos são também organismos, personalidades e participantes de sistemas culturais.

Parsons (1974) destaca, ainda, que os sistemas sociais são os constituintes do sistema mais geral de ação; os demais componentes são os sistemas culturais (que mantém um padrão); os sistemas de personalidade (que busca a realização dos objetivos) e organismo comportamental (adaptação e de relação com o ambiente físico). Os sistemas sociais são considerados abertos, participando de um intercâmbio contínuo de recepções e apresentações com seus ambientes.

Morgan (1996) define organizações como organismos, as quais são concebidas como sistemas vivos que existem em um ambiente mais amplo do qual dependem em termos de satisfação das suas várias necessidades. O autor destaca o enfoque dos sistemas abertos que gerou conceitos novos ao pensar nas organizações, visto que as organizações são sistemas orgânicos que existem num processo contínuo de trocas com seus ambientes, o qual é crucial para a manutenção da vida e forma do sistema. Algumas características adicionais são: 1) Homeostase: Autorregulamentação e a capacidade de conservar um estado equilibrado; 2) Entropia: Característica dos sistemas fechados, uma vez que tem a tendência de se deteriorar e parar. Os sistemas abertos, por outro lado, tentam sustentar-se, importando energia para tentar compensar as tendências entrópicas; 3) Estrutura, função, diferenciação e integração: 0 relacionamento entre esses conceitos é de importância crucial para compreender os sistemas vivos; 4) Variedade de requisito: Somente pela incorporação da requerida variedade aos seus controles internos, que o sistema pode interagir com a variedade e o desafio propostos pelo seu ambiente. Qualquer sistema que se isola da diversidade do ambiente tende a atrofiar-se e a perder a sua complexidade bem como a distintividade da sua natureza. Assim, o requisito variedade é uma importante característica dos sistemas vivos; 5) Equifinalidade: Sistemas vivos possuem padrões flexíveis de organização que permitem atingir resultados específicos a partir de diferentes pontos de partida, com diferentes recursos e maneiras; 6) Evolução do sistema: A possibilidade que tem um sistema de evoluir depende de sua capacidade de passar a formas de diferenciação e integração mais complexas, bem como a uma variedade maior no sistema que facilite a sua habilidade em lidar com desafios e oportunidades propostos pelo ambiente.

Morgan (1996) destaca, também, a teoria contingencial como a adaptação da organização ao ambiente. Neste enfoque, a organização necessita de uma administração que satisfaça e equilibre as necessidades internas, assim como se adapta a circunstâncias ambientais. Esta administração deve estar preocupada acima de tudo em atingir boas medidas, e neste processo diferentes tipos de organização são necessários em diferentes tipos de ambiente. Assim se faz necessário um enfoque mais orgânico e flexível do que um aspecto mecanicista de pouca adaptação ao ambiente.

A teoria geral dos sistemas pode ser capaz de contribuir com o desenvolvimento organizacional. É valioso considerar porque a teoria de sistemas pode avançar: Primeiramente,

Perspectivas em Gestão \& Conhecimento, João Pessoa, v. 7, n. 1, p. 191-211, jan./jun. 2017. 
assume-se que sistemas complexos como organizações são caracterizados como universalmente caracterizados por importantes feedbacks (retroalimentação) que mantém a estabilidade do sistema. Quando o indivíduo deixa o ciclo, a organização busca um substituto. Comportamentos divergentes devem ser corrigidos. Atividades de sucesso devem ser encorajadas. Sistemas complexos são constantemente atacados por forças internas e externas que destruiriam o funcionamento do sistema ao menos que houvesse uma reação. A reação é estimulada pelo desenvolvimento organizacional (PONDY; CONNOLLY, 1989).

Muitas organizações encontram sérios problemas em lidar com o mundo exterior por não reconhecerem que são parte dos seus respectivos ambientes. Consideram-se como entidades isoladas que se deparam com o problema de sobreviver contra os caprichos do mundo exterior que é concebido como um lugar de ameaças e oportunidades. Estas são organizações consideradas como egocêntricas que ficam preocupadas com elas mesmas e que superenfatizam a própria importância, ao mesmo tempo em que subestimam o significado do sistema mais amplo de relações que existem ao seu redor (MORGAN, 1996).

Quando se reconhece que o ambiente não se trata de um campo independente e que não há como lutar ou competir com o ambiente, um relacionamento completamente novo se torna possível. Organizações comprometidas com este relacionamento são capazes de desenvolver um tipo de sabedoria sistêmica. Tornam-se mais conscientes do seu papel, do seu significado dento do todo e de sua habilidade de facilitar padrões de mudança e de desenvolvimento que permitirão a evolução conjunta com o sistema mais amplo (MORGAN, 1996).

Neste contexto, Parsons (1966) destaca que entre os processos de mudança, o tipo mais importante para a perspectiva do desenvolvimento evolutivo é o aumento de capacidade adaptativa, seja quando no interior da sociedade surge uma nova estrutura, seja quando esta nova estrutura surge em outra sociedade, ou por meio da difusão cultural e da interferência de outros fatores combinados a esta nova estrutura.

Parker (1999) salienta que a adaptação da estrutura organizacional à globalização requer não só mudanças incrementais no funcionamento da organização, mas um repensar fundamental como os participantes da organização pensam seu relacionamento com a organização e o papel da organização num mundo global.

Já Hall (2004), destaca a importância crítica dos ambientes organizacionais, na qual expressões como economia global em mercados centrais e periféricos ressaltam o fato de que todas as organizações são afetadas por seu entorno. O ambiente penetra na organização sob a forma de informação, a qual está sujeita a problemas de comunicação e tomada de decisões que foram identificados. Informações ambientais são informações a serem processadas.

Assim, a informação se torna um requisito fundamental no processo de relacionamento com o ambiente externo, para que seja possível a aprendizagem e mudança organizacional.

Neste ambiente, onde as organizações necessitam obter lucratividade suficiente para atuar competitivamente no mercado, se faz necessário à adoção de uma série de estratégias pela empresa, que possuam o poder de conferir capacidade produtiva às atividades desenvolvidas pela empresa. Para subsidiar estas estratégias ressalta-se que o uso de informações (internas ou externas) contribui para a agilidade das tomadas de decisões empresariais já que fornecem dados sobre o desempenho organizacional (SANTOS et al, 2013)

\subsection{Gerenciamento de Riscos Corporativos (GR): Conceituação}

Conforme Cocurullo (2004), risco é a possibilidade de algo não dar certo ou um imprevisto mudar o rumo dos acontecimentos. O risco é inerente a qualquer atividade, decisão e até à própria vida pessoal, profissional e de qualquer entidade viva. A natureza humana 
interpreta o risco mais por seu lado negativo, no entanto, decisões envolvendo altos riscos podem também gerar efeitos altamente positivos (oportunidades).

Oportunidade é a possibilidade de que um evento ocorra e influencie favoravelmente a realização dos objetivos (COSO, 2007).

Neste contexto, Padoveze (2010) classifica o conceito de risco nas perspectivas abaixo:

- Risco como oportunidade: Implícito no conceito de risco e retorno. Quanto maior o risco, maior o potencial de retorno, e necessariamente, de perda. Neste contexto, a gestão de riscos significa utilizar técnicas para maximizar as oportunidades e minimizar os riscos.

- Risco como perigo ou ameaça: Refere-se a eventos potencialmente negativos como: perdas financeiras, fraudes, danos à reputação, roubo ou furto, morte ou injúria, falhas de sistemas ou demandas judiciais. Neste cenário, a gestão de riscos significa implantar ações administrativas para reduzir a probabilidade de eventos negativos sem incorrer em custos excessivos ou paralisar a organização.

- Risco como incerteza: Relacionado à distribuição de todos os resultados possíveis, sejam positivos ou negativos. Neste panorama, a gestão de risco procura reduzir a variância entre os resultados planejados versus reais.

Já o foco e a preocupação com o gerenciamento de riscos foi intensificada nos últimos anos (principalmente motivada por escândalos de fraudes e quebras de negócio no início do século XXI), e tornou-se cada vez mais clara a necessidade de uma estratégia sólida, capaz de identificar, avaliar e administrar riscos. O cOSO-Committee of Sponsoring Organizations of the Treadway Commission (2007) define que:

O gerenciamento de riscos corporativos é um processo conduzido em uma organização pelo conselho de administração, diretoria e demais empregados, aplicado no estabelecimento de estratégias, formuladas para identificar em toda a organização eventos em potencial, capazes de afetá-la, e administrar os riscos de modo a mantê-los compatível com o apetite a risco da organização e possibilitar garantia razoável do cumprimento dos seus objetivos (COSO, 2007, p. 4).

A premissa inerente ao GR é que toda organização existe para gerar valor às partes interessadas, no entanto, as organizações enfrentam incertezas, e o desafio de seus administradores é determinar até que ponto aceitar essas incertezas que representam riscos e oportunidades, com potencial para destruir ou agregar valor (COSO, 2007).

Um modelo de GR possibilita aos administradores tratar com eficácia as incertezas, bem como os riscos e as oportunidades a elas associadas, a fim de melhorar a capacidade de gerar valor as partes interessadas. $O$ valor é maximizado quando a organização estabelece estratégias e objetivos para alcançar o equilíbrio ideal entre as metas de crescimento e de retorno de investimentos e os riscos a elas associados (COSO, 2007).

Dentro deste conceito de equilíbrio, Padoveze (2010) destaca que o foco da GR é manter um processo sustentável de criação de valor para os acionistas, uma vez que qualquer negócio está constantemente exposto aos riscos.

Por outro lado, Coso (2007) destaca que o GR contribui para assegurar comunicação eficaz dos eventos adversos e o cumprimento de leis e regulamentos, bem como evitar danos à reputação da organização e suas consequências. Desta forma, este mecanismo ajuda a organização a atingir seus objetivos e a evitar os perigos e surpresas em seu percurso. 


\subsection{Gerenciamento de Riscos Corporativos (GR) e Controladoria}

Ben-Amar et al. (2014) definem que os conceitos de Estratégia e Risco são extremamente relacionados na teoria e na prática. Ambos constituem a base para as decisões dentro das companhias. Riscos são inerentes a qualquer escolha estratégica dentro das empresas. Assim, o GR é uma abordagem rigorosa que analisa todos os riscos da companhia que pode levar ao não atingimento dos objetivos estratégicos.

Dentro deste enfoque, a área de Controladoria passa a ser um dos principais estimuladores e pontos de controle do processo de GR. A importância da Controladoria foi reforçada com sua nova perspectiva, denominada Controladoria Estratégica, que leva esta área a colaborar desde o planejamento estratégico, execução e controle da operação.

Assim, a Controladoria é um importante organismo de apoio ao planejamento e controle nas organizações. Mosimann e Fisch (2008) destacam que a Controladoria deve suportar a organização com a coordenação de sistema de informações econômico-financeiras que envolve todo o processo de gestão econômica: Planejamento dos eventos econômicos futuros; Elaboração de padrões para a etapa de execução; Implementação das ações planejadas (execução); Controle, por meio da comparação entre o executado e o planejado. Assim, a Controladoria busca a otimização do resultado econômico global para a continuidade da empresa e o atingimento de sua missão.

Este foco é reforçado por Padoveze (2012) que destaca que o lucro é a medida de eficácia da empresa, assim a Controladoria deve buscar a gestão com base em resultados. Todos os esforços empresariais e a missão da Controladoria devem ter como ponto referencial o processo de criação de valor para o acionista, por meio da mensuração econômica do valor da empresa. O autor complementa as áreas de atuação estratégica do controller (principal executivo da Controladoria):

- No processo de mensuração dos resultados corretos das atividades empresariais, permitindo à empresa a tomada de decisão adequada.

- No seu papel influenciador, pelo apoio que presta às demais atividades dentro da empresa, na sua missão como assegurador da eficácia empresarial.

- Ser o responsável pelos sistemas de informações abastecedores do Planejamento Estratégico e do controle das metas estratégicas. O Sistema de Informação Contábil deve ser estruturado para atender as necessidades informacionais de todo o processo de gestão da empresa. Desde a etapa do Planejamento Estratégico até o processo final de controle, passando pela programação, o sistema contábil deve estar apto para municiar e receber informações operacionais e financeiras.

Este papel de responsável pelos sistemas de informações citado por Padoveze é importante e dá destaque a área de Controladoria, pois conforme Pereira (2001) o processo de gestão constitui-se num processo decisório que requer informações.

Os sistemas de informações devem apoiar as decisões dos gestores em todas as fases do processo de gestão (requerem informações específicas). Os sistemas de informações integrados ao processo de gestão determinam a eficácia dos mecanismos de autocontrole e feedback que são requisitos para que a empresa busque resultados desejados (PEREIRA, 2001).

Em seu livro que aborda sobre Controladoria Estratégica, Padoveze (2012) estrutura o modelo demonstrado na Figura 1 partindo da premissa que a organização assume a necessidade de: Sistema integrado de gestão (ERP), que possua a característica de unir e integrar todos os subsistemas operacionais e de apoio à gestão; Data Warehouse (DW) para

Perspectivas em Gestão \& Conhecimento, João Pessoa, v. 7, n. 1, p. 191-211, jan./jun. 2017. 
armazenar todos os dados que são de interesse da média administração, originários dos sistemas operacionais e de apoio à gestão, incluídos no ERP e fontes externas à organização; e Business Intelligence (BI) para oferecer as informações produzidas a partir do tratamento de grandes volumes de dados que se encontram no DW. A depender da quantidade de dados tratados pela organização subsistemas BI e DW podem ser unificados.

No ambiente estratégico, segundo o modelo proposto na Figura 1, o sistema de informação de Controladoria Estratégica é subdividido em quatro sistemas:

- Cenários empresariais: informações do mercado, concorrência, economia.

- Sistema de acompanhamento de negócios: Compara dados internos e externos e projetam seus impactos no orçamento da organização.

- Balanced Scorecard (BSC): monitoramento dos indicadores chaves de desempenho os quais são desdobrados a partir da estratégia da organização.

- Gestão de riscos: Identificação, Mensuração e Controle das ameaças e oportunidades que podem afetar a consecução dos objetivos.

Figura 1 - Sistemas de informações de Controladoria Estratégica

\begin{tabular}{|c|c|c|c|}
\hline $\begin{array}{c}\text { Cenàrios } \\
\text { Empresariais }\end{array}$ & $\begin{array}{l}\text { Sistema de } \\
\text { Acompanhamento } \\
\text { do Negócio }\end{array}$ & $\begin{array}{l}\text { Indicadores de } \\
\text { Desempenhoe } \\
\text { Balanced } \\
\text { Scorecard } \\
\end{array}$ & Gestăo de Riscos \\
\hline \multicolumn{4}{|c|}{$\begin{array}{l}\text { Estratégico - BI (Business Intelligence) } \\
\text { Oferecer informacôes produzidas a partir do tratamento de grandes volumes de dados que se } \\
\text { encontram no Data Warebouse. }\end{array}$} \\
\hline \multicolumn{4}{|c|}{$\begin{array}{l}\text { Tático - DW (Data Warehouse) } \\
\text { Armazenar dados que sảo de interesse da média administracio, originairios dos sistemas operacionais } \\
\text { de apojo a gestio, constantes do ERP, e mesmo de fontes esternas a organizaçio. }\end{array}$} \\
\hline \multicolumn{4}{|c|}{$\begin{array}{c}\text { Operacional - ERP (Enterprise Resources Planning) } \\
\text { Tem a caracteristica de unir e integar todos os subsistemas componentes dos sistemas operacionais } \\
\text { e dos sistemas de apoio a gestio. }\end{array}$} \\
\hline
\end{tabular}

Fonte: Adaptado de PADOVEZE (2012, p. 101).

Nascimento et al. (2007) também reforçam que a Controladoria deve antecipar a análise dos impactos ambientais, sendo necessária, às vezes uma revisão do processo de gestão, por meio do planejamento estratégico e operacional. Assim, a empresa poderá se programar para que prossiga na sua missão de forma sólida.

Já Lira et al. (2012) abordam que gerenciar organizações em um ambiente de negócios dinâmico é um desafio constante, no qual o ciclo de vida dos produtos e serviços tem diminuído devido a crescente evolução tecnológica. Empresas visam aprimorar continuamente seus processos de negócios para a execução de seus planos. Neste contexto, a Controladoria no sistema da companhia deve captar influências internas e externas, especialmente no que se refere a mudanças tecnológicas e globalização de processos interconectados.

Assim, a Controladoria se firma como um grande parceiro das organizações para auxilia-las a identificar e se adaptar a esta nova realidade. Para atender esta incumbência, a Controladoria pode aprimorar a eficiência e eficácia organizacional por meio do uso da Tecnologia de Informação realizando atividades divididas em 2 grandes grupos:

- Controles Gerenciais: Objetiva assegurar a eficácia organizacional por meio de um processo estruturado de gerenciamento composto de planejamento, execução e controle. 
- Controles Operacionais: Visa assegurar a execução eficiente de atividades operacionais resultantes das decisões tomadas, por meio do uso ótimo de recursos promovendo salvaguarda dos ativos (LIRA et al, 2012).

Lira et al. (2012) acrescentam, ainda, que o uso de sistemas integrados de gestão (ERP - Enterprise Resources Planning) apoiado pela Controladoria possuem impactos significativos para o processo de Gerenciamento de Riscos e Controles Internos, tais como:

a) Controle de processos integrados: Delimitação de funções e atividades em diversas áreas, reagrupando ou redistribuindo, no caso de mudanças organizacionais ou mesmo complexas reestruturações.

b) Redesenho de processos: Padronização de rotinas e processos, o que habilita uma revisão de processos baseada em boas práticas, testadas e operacionalizadas em outras companhias. Esta prática auxilia na execução ótima dos processos.

c) Compliance: Aderência a requerimentos legais: Cumprimento de requerimentos legais como Fiscais.

Nesta linha, Brito (2003) conceitua que a Controladoria procura avaliar o risco de controle interno. Este risco se materializa quando há falta de consistência e adequação dos sistemas de controle interno acarretando em perdas inesperadas para a empresa. Para melhor execução desta atividade, a Controladoria incorpora novas dimensões no universo do controle do risco migrando da análise do retorno obtido para análise do retorno em condições de risco e alocação de capital. Assim, a Controladoria assume uma tridimensionalidade:

a) Coleta de dados: Dados a respeito do uso de recursos (custos e receitas) e a respeito do risco na utilização de recursos;

b) Preparação dos demonstrativos financeiros: Devem incorporar medidas de risco, ao lado das medidas de rentabilidade; e

c) Análise de desempenho: Devem prover medidas simultâneas bidimensionais de retorno e risco em cada produto e linha operacional.

Dentro desta vertente, a Controladoria estende suas funções clássicas (como controle de custos, controle e acompanhamento orçamentário, sistemas gerenciais, relatórios para autoridades regulatórias e contabilidade societária) para incorporar o controle dos riscos por meio de sua identificação, mensuração e divulgação focando a comparação retorno e risco (BRITO, 2003).

Assim, cabe à Controladoria contribuir para que o sistema de controles internos da empresa seja sólido e robusto, a fim de manter as boas práticas de governança corporativa, seja, elas fruto de imposição legal ou não (NASCIMENTO et al., 2007).

\section{PROCEDIMENTOS METOdOLÓGICOS}

Esta pesquisa adota a abordagem qualitativa, ou seja, o paradigma interpretativo para análise dos resultados, contemplado pela abordagem quantitativa (paradigma funcionalista) para quantificação dos dados.

Conforme Godoy (1995) destaca que as características básicas da pesquisa qualitativa são: (1) tem o ambiente natural como fonte direta de dados e o pesquisador como instrumento fundamental; (2) ser uma pesquisa descritiva que possui uma compreensão ampla

Perspectivas em Gestão \& Conhecimento, João Pessoa, v. 7, n. 1, p. 191-211, jan./jun. 2017. 
do fenômeno que está sendo estudado e considera que todos os dados da realidade são importantes e devem ser examinados; (3) o significado que as pessoas atribuem às coisas e à sua vida são a preocupação essencial do investigador; (4) pesquisadores utilizam o enfoque indutivo na análise de seus dados. Por outro lado Shah e Corley (2006) destacam que o que é importante é a interpretação das experiências de um fenômeno em estudo, no qual cada pessoa que conduz a pesquisa possui uma interpretação única dos resultados. A pesquisa qualitativa pode complementar uma análise quantitativa. (SHAH; CORLEY, 2006).

Os procedimentos definidos na investigação empírica são a pesquisa bibliográfica e pesquisa exploratória e tiveram como objetivo mapear a produção científica no tema Riscos envolvendo o objeto de estudo, a amostragem e a forma de coleta de dados, além do modo de operacionalização das variáveis.

Trata-se de um estudo bibliométrico. Os teóricos e pesquisadores da bibliometria desenvolveram e criaram "Leis" específicas para a análise da produção cientifica. Em Chen et al(1994), as três leis de distribuição bibliométrica mais conhecidas nesta disciplina são: a Lei de Lotka, Lei de Zipf e Lei de Bradford. Vanti (2002) define cada uma das leis conforme segue: (I) Lei de Lotka, ou Lei do Quadrado Inverso, aponta para a medição da produtividade dos autores, mediante um modelo de distribuição tamanho-frequência dos diversos autores em um conjunto de documentos. (2) Lei de Zipf, ou Lei do Mínimo Esforço, consiste em medir a frequência do aparecimento das palavras em vários textos, gerando uma lista ordenada de termos de uma determinada disciplina ou assunto. (3) Lei de Bradford, ou Lei de Dispersão, permite, mediante a medição da produtividade das revistas, estabelecer o núcleo e as áreas de dispersão sobre um determinado assunto em um mesmo conjunto de revistas. Assim, pode-se afirmar que para a consecução dos objetivos desta pesquisa utilizou-se da Lei de Zipf e Bradford.

A complexidade do tema GR corroborou para a escolha do objeto de pesquisa. Assim, a contribuição deste estudo se faz importante na medida em que amplia a reflexão atual das tendências do tema permitindo o avanço cientifico.

Para identificar as tendências das pesquisas realizou-se uma análise bibliográfica na Base de Dados Scopus, uma das maiores bases de dados de resumos e citações da literatura. Oferece uma visão abrangente da produção de pesquisas do mundo nas áreas de ciência, tecnologia, medicina, ciências sociais e artes e humanidades. A escolha da Base de Dados Scopus se deu também em virtude da disponibilização dos resumos, além dos títulos e palavras-chave.

A pesquisa foi realizada durante o mês de 25 de Maio de 2015 a 12 de Junho de 2015. Inicialmente realizou-se uma busca nas palavras-chave, no resumo e título das publicações envolvendo a palavra Risco. Antes do processo de análise no VOSviewer demandou-se a escolha dos parâmetros de corte. Estratificou-se uma amostra não probabilística e intencional por conveniência, O Quadro 1 demonstra as áreas, palavras, países e períodos de corte:

\begin{tabular}{|l|l|}
\multicolumn{1}{|c|}{ Quadro 1 - Parâmetros de corte da pesquisa } \\
\hline Parâmetro & \multicolumn{1}{c|}{ Descrição } \\
\hline Palavras-chave & $\begin{array}{l}\text { Selecionou-se apenas a produção de artigos da área de Economia, Econometria e } \\
\text { Finanças, Negócios, Gestão e Contabilidade. Tais áreas foram selecionadas pois } \\
\text { entende-se que são mais aderentes ao objetivo da pesquisa que é identificar } \\
\text { pesquisas sobre o papel da Controladoria na estruturação de sistemas de } \\
\text { informação para GR. }\end{array}$ \\
\hline Países & $\begin{array}{l}\text { Envolvendo a palavra Riscos. Avaliação de Riscos, Gerenciamento de Riscos, Risco, } \\
\text { Fator de Risco, Percepção de Risco, Aversão a Risco, Análise de Riscos. }\end{array}$ \\
\hline
\end{tabular}

Perspectivas em Gestão \& Conhecimento, João Pessoa, v. 7, n. 1, p. 191-211, jan./jun. 2017. 


\begin{tabular}{|l|l|}
\hline & $\begin{array}{l}\text { número de publicações sobre o tema (cerca de 40\%), bem como é um país que } \\
\text { apresentou forte abordagem sobre o tema devido aos escândalos corporativos } \\
\text { ocorridos no início do século (como envolvendo as empresas Enron e WorldCom), } \\
\text { tal como a crise financeira do ano de } 2008 \text { (dos papéis tóxicos imobiliários } \\
\text { chamados subprimes) que culminou com uma grave crise mundial. } \\
\text { Já a escolha do Brasil, se deve ao fato do local onde a pesquisa está sendo } \\
\text { realizada e aos recentes escândalos corporativos envolvendo operação Lava Jato } \\
\text { que tem como objetivo principal a investigação do desvio de recursos públicos da } \\
\text { Petrobrás. Estes escândalos destacaram a necessidade das práticas de governança, } \\
\text { riscos e compliance das empresas brasileiras. }\end{array}$ \\
\hline $\begin{array}{l}\text { Período } \\
\text { pesquisa }\end{array}$ & de a 2015. Com objetivo de se pesquisar artigos mais atuais sobre o tema. \\
\hline
\end{tabular}

Fonte: Elaborado pelos autores

Posteriormente os dados foram compilados, exportados (em formato *.ris) para serem analisados e se tornarem informações úteis.

Desenvolveu-se uma aplicação do uso do software VOSviewer baseada na análise dos corpos dos textos. O software desenvolve um processo de clusterização por meio da implementação de um algoritmo de mapeamento - VOS - que busca minimizar a distância Euclidiana entre elementos semelhantes e destina-se principalmente à análise bibliométrica de grandes volumes de dados dando ênfase na representação gráfica do mapa (RESCH e FARINA, 2014).

O software utiliza uma medida de semelhança conhecido como a força de associação que deve ser compreendido como representações de escalonamento multidimensional direto (VAN ECK; WALTMAN, 2007). Para Peters e Van Raan (1993); Rip e Courtial (1984) esta medida de similaridade às vezes é referida como o índice de proximidade ou como o índice de afinidade probabilística (Zitt et al, 2000). Segundo Van Eck e Waltman (2007) o VOSviewer utiliza a força de associação, o Sij representa a semelhança entre dois itens i e j, calculado como:

$$
s_{i j}=\frac{c_{i j}}{w_{i} w_{j}},
$$

O Cij indica o número de coocorrências dos itens i e j e wi, wj representam ou o número total de ocorrências dos itens i e j ou o número total de coocorrências desses itens. Demonstrou-se que a semelhança entre os itens i e j calculada, usando (1), é proporcional à relação entre o número observado de coocorrências dos incisos i e j e sob a suposição de que as ocorrências de itens i e j são estatisticamente independentes. Para evitar mapas triviais em que todos os itens têm a mesma localização, o software utiliza como restrição imposta a distância média entre dois itens igual a 1 . A equação matemática é a seguinte:

$$
V\left(\mathbf{x}_{1}, \ldots, \mathbf{x}_{n}\right)=\sum_{i<j} s_{i j}\left\|\mathbf{x}_{i}-\mathbf{x}_{j}\right\|^{2},
$$

$\mathrm{O}$ vetor $\mathrm{xi}=(\mathrm{xi} 1, \mathrm{xi2})$ indica a localização do item i num mapa bidimensional onde indica $\|\bullet\|$ a norma euclidiana. Face a minimização da função objetivo é executada sujeito à restrição:

$$
\frac{2}{n(n-1)} \sum_{i<j}\left\|\mathbf{x}_{i}-\mathbf{x}_{j}\right\|=1 .
$$

Assim o problema de otimização obrigados de minimizar (2) sujeito a (3) é resolvido numericamente em duas etapas. Van Eck e Waltman (2007) demonstram que o problema de otimização constrangido é convertido primeiro em um problema de otimização sem restrições.

Perspectivas em Gestão \& Conhecimento, João Pessoa, v. 7, n. 1, p. 191-211, jan./jun. 2017. 
A densidade do produto de um ponto $X$ para um grupo $p$, denotado por $\mathrm{Pd}(\mathrm{X})$, é definido como:

$$
D_{p}(\mathbf{x})=\sum_{i=1}^{n} I_{p}(i) w_{i} K\left(\left\|\mathbf{x}-\mathbf{x}_{i}\right\| /(\bar{d} h)\right)
$$

A partir do (4) edificou-se as relações e correlações dos clusters. Quanto maior for o número de vizinhos e as menores distâncias entre os itens e o ponto de interesse, maior a densidade do produto. Além disso, quanto maior for o peso dos itens adjacentes, maior o item densidade (VAN ECK; WALTMAN, 2007). Assim, o cálculo das densidades é semelhante à estimativa de uma função de densidade de probabilidade usando a técnica de estimação de densidade de granulação.

Após o cálculo dos scores de densidade, selecionaram-se os termos, dentre os mais recorrentes, mais relevantes para a pesquisa, a fim de mapear o conhecimento sobre o papel da Controladoria na estruturação de sistemas de informação para GR, evidenciando-se as similaridades e diferenças terminológicas, considerando-se os reflexos dos efeitos axiológicos, existentes.

A Figura 2 demonstra um sumário dos passos da pesquisa:

Figura 2 - Etapas para obtenção dos dados

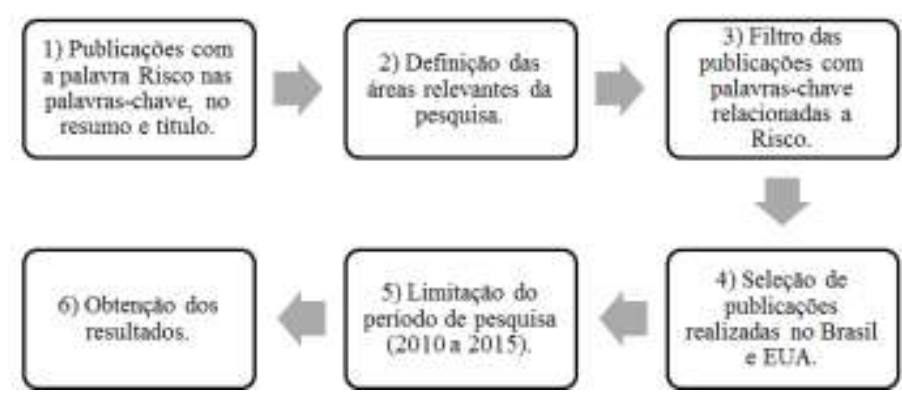

Fonte: Elaborado pelos autores

Esta figura demonstra as etapas realizadas para seleção e filtro dos dados com objetivo de levar aos resultados da pesquisa que são demonstrados na seção a seguir.

\section{ANÁLISE DOS RESULTADOS}

Os resultados da pesquisa na Base de Dados SCOPUS revelam que o número de pesquisas envolvendo Riscos (nas palavras-chave, no resumo e título) totalizou 2.198.712 nos últimos sete anos.

A classificação dos artigos por áreas evidenciou que há uma grande quantidade de pesquisas nas áreas de Medicina (1.637.077), Bioquímica, Genética e Biologia Molecular (268.150) e Ciências Sociais (112.940). Para análise pormenorizada, prosseguiu-se com a seleção da área de Economia, Econometria e Finanças (41.434), Negócios, Gestão e Contabilidade (34.309). Os filtros das palavras chaves citadas no quadro 1 levou a quantidade de 13.091.

Posteriormente, foram selecionados os países: EUA, cujo teve o maior número de publicações (4.524); e Brasil (121) local onde a pesquisa está sendo realizada. Finalmente, foi filtrado o período de 2010 a 2015, assim obtivemos o número de 1.714 artigos sendo 1.638

Perspectivas em Gestão \& Conhecimento, João Pessoa, v. 7, n. 1, p. 191-211, jan./jun. 2017. 
artigos nos EUA e 87 no Brasil. Os dados foram tratados no software VOSviewer e conforme a Figura 3 foram demonstrados os termos em destaque:

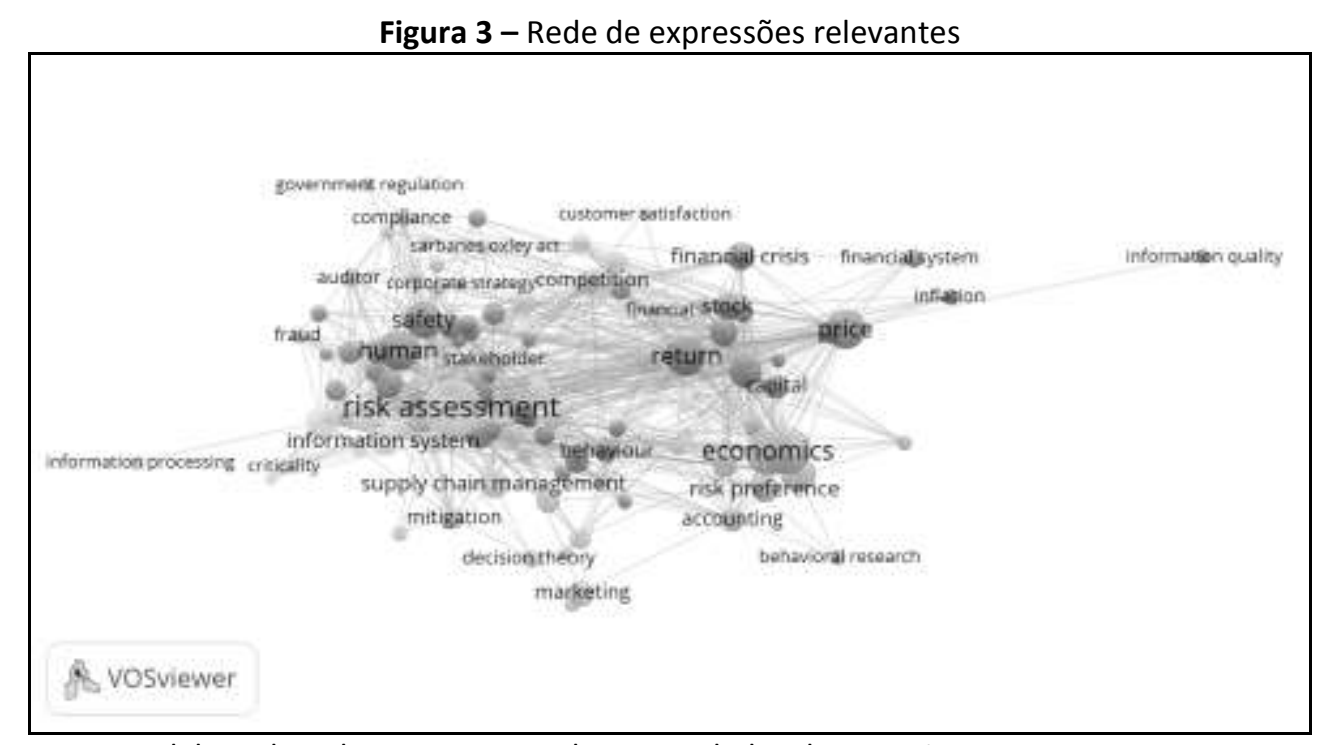

Fonte: Elaborado pelos autores com base nos dados da pesquisa

O VOSviewer apresenta as palavras que tem maior densidade com destaque em vermelho. Observa-se assim, por meio da Figura 4, que as expressões Avaliação de Riscos e Economia foram os termos mais recorrentes nas pesquisas da área.

Figura 4 - Densidade da coocorrência das palavras

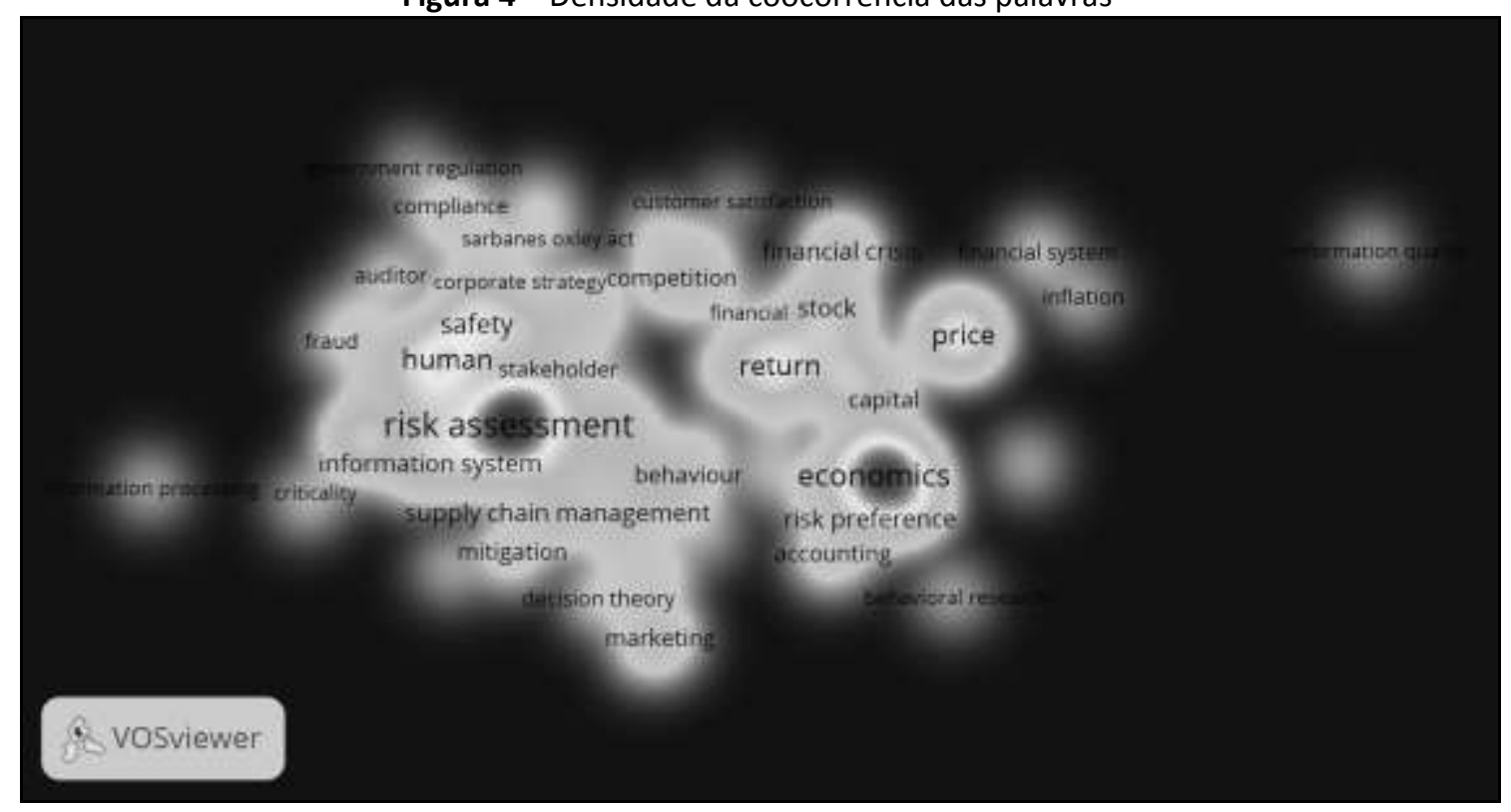

Fonte: Elaborado pelos autores com base nos dados da pesquisa

Observam-se algumas características relevantes:

- Não há uma relação entre qualidade da informação, avaliação de riscos e sistemas de informação. 
- A palavra processo não é evidenciada. Entende-se que o GR é formado por um tripé (Pessoas, Processos e Tecnologia) e deveria existir uma relação entre GR e este tripé em pesquisas da área.

- A palavra Controladoria não foi evidenciada, o que demonstra a ausência de pesquisas que relacionam Controladoria e GR.

- Não há uma relação entre os temas GR, Governança Corporativa e Gestão Baseada em Valor, visto que Governança Corporativa não possui destaque e a Gestão Baseada em Valor não foi evidenciada.

Já o Quadro 2 apresenta a classificação dos clusters:

Quadro 2 - Clusters das palavras com maior destaque na pesquisa

\begin{tabular}{|c|c|c|c|}
\hline Cluster 1 & Cluster 2 & Cluster 3 & Cluster 4 \\
\hline Acceptance & Competition & Behaviour & Criticality \\
\hline Collaboration & conceptual framework & $\begin{array}{l}\text { corporate social } \\
\text { responsibility }\end{array}$ & Database \\
\hline conceptual model & corporate strategy & crisis management & entrepreneurship \\
\hline Culture & cost benefit analysis & Disruption & Evaluation \\
\hline Employment & customer satisfaction & Globalization & Globalisation \\
\hline Energy & Diversification & information management & information processing \\
\hline $\begin{array}{l}\text { enterprise risk } \\
\text { management }\end{array}$ & Financial & information sharing & information system \\
\hline Ethic & $\begin{array}{l}\text { human resource } \\
\text { management }\end{array}$ & reputation & information technology \\
\hline Guideline & Identification & risk communication & management practice \\
\hline Human & Involvement & risk research & Resilience \\
\hline information security & Marketing & supply chain & risk assessment \\
\hline Leadership & perceived risk & supply chain disruption & risk mitigation \\
\hline Models & project risk management & supply chain management & Training \\
\hline $\begin{array}{l}\text { organizational } \\
\text { performance }\end{array}$ & risk control & supply chain network & \\
\hline performance evaluation & $\begin{array}{l}\text { risk management } \\
\text { framework }\end{array}$ & & \\
\hline Prevention & risk management practice & & \\
\hline risk measure & Stakeholder & & \\
\hline \multicolumn{4}{|l|}{ Safety } \\
\hline \multicolumn{4}{|l|}{ Treatment } \\
\hline Cluster 5 & Cluster 6 & Cluster 7 & Cluster 8 \\
\hline Bank & Auditor & accounting & decision theory \\
\hline Capital & business process & asymmetric information & economic analysis \\
\hline Economy & Compliance & asymmetry & Integration \\
\hline Equity & corporate governance & behavioral research & international trade \\
\hline financial crisis & Fraud & economics & Mitigation \\
\hline financial system & Governance & finance & Partnership \\
\hline global financial crisis & government regulation & information asymmetry & risk management strategy \\
\hline Inflation & internal control & relative risk aversion & $\begin{array}{l}\text { supply chain risk } \\
\text { management }\end{array}$ \\
\hline information quality & Inventory & risk averse & technological innovation \\
\hline Price & operational risk & risk aversion & Transparency \\
\hline Return & sarbanes oxley act & risk preference & \\
\hline \multicolumn{4}{|l|}{ Stock } \\
\hline systemic risk & & & \\
\hline
\end{tabular}

Fonte: Elaborado pelos autores com base nos dados da pesquisa 
Com relação à classificação de clusters, notou-se que:

- O cluster 6 que aborda sobre Governança Corporativa, Compliance e Controles Internos não está no mesmo agrupamento que GR ou Avaliação de Riscos (cluster 1, 2 e 4), o que suporta que os assuntos não estão conectados nas pesquisas.

- Os temas GR (cluster 1, 2 e 4), Recursos Humanos (cluster 2), Sistemas de Informação (cluster 4) e Processos de Negócios (cluster 6) também não estão relacionados nas pesquisas.

- Não se evidencia o tema Controladoria ou Contabilidade Gerencial em nenhum dos clusters.

Assim, tais resultados demonstram lacunas relevantes existentes envolvendo a pesquisa de Gerenciamento de Riscos Corporativos e sua interligação com a Controladoria e a estruturação de sistemas de informações.

\section{CONSIDERAÇÕES FINAIS}

A pesquisa objetivou demonstrar a importância do tema GR e estabelecer sua interligação com a Controladoria, a qual pode suportar a estruturação de sistemas de informação que alimentem este processo de gestão.

Dentro da complementaridade do tema, abordou-se sobre a conexão direta entre GR e boas práticas de gestão como a Governança Corporativa que vem sendo cada vez mais exigidas de todas as companhias, principalmente das de capital de aberto.

Diante deste cenário evolutivo e complexo, destaca-se a necessidade de aprofundamento da temática envolvendo GR e suas práticas que podem aprimorar a sua execução dentro das organizações.

Entende-se que o problema de pesquisa foi respondido pela identificação das pesquisas que vem sendo realizadas quanto à estruturação de sistemas de informação para $\mathrm{GR}$, as quais estão focadas na avaliação de riscos e aspectos relacionados à economia, preço e retorno.

Por outro lado, este trabalho demonstrou importantes lacunas referentes ao tema GR e a participação da área de Controladoria neste processo. As lacunas observadas neste estudo bibliométrico apontam para o não relacionamento de Gerenciamento de Riscos, Governança Corporativa, Compliance e Controladoria na amostra pesquisada.

Ademais, não há uma relação direta entre GR e o tripé do processo de gestão de qualquer companhia e processo de negócio: Pessoas, Processos e Tecnologia. Por melhor que sejam os processos e tecnologias implementados por qualquer organização, caso as pessoas não estejam preparadas, os objetivos estipulados se tornaram mais distantes de serem alcançados.

Possivelmente, este resultado se deve ao baixo número de pesquisas sobre GR e práticas relacionadas à estruturação de sistemas de informação e processos de administração de riscos. Apesar de este tema ser uma preocupação crescente das organizações, seus administradores e órgãos regulatórios, as pesquisas acadêmicas sobre estes temas necessitam evoluir para endereçar possibilidades de melhoria destas práticas.

Portanto, este resultado demonstra a ausência de pesquisas sobre elementos intimamente ligados à estruturação de sistemas de informação para GR e consequentemente sugere-se o aprofundamento das pesquisas nesta área, principalmente, as que se relacionarem com sua integração a Controladoria, bem como abordando sobre a importância das pessoas e processos de negócio neste tema.

Perspectivas em Gestão \& Conhecimento, João Pessoa, v. 7, n. 1, p. 191-211, jan./jun. 2017. 
Quanto aos procedimentos metodológicos, a análise bibliométrica por meio de uma conceituada base de dados como a SCOPUS e a utilização do software VOSviewer, demonstra um importante método para identificação das áreas de conhecimento pesquisadas sobre determinado tema e possibilidades existentes para novas pesquisas. Portanto, sendo um mecanismo disponível para construção do conhecimento e consequentemente aprimoramento de práticas em áreas de atuação como Gestão, Finanças e Contabilidade.

Como limitações desta pesquisa destacam-se: a comparação das pesquisas entre Brasil e EUA envolvendo a temática Riscos. Apesar dos EUA tratar-se do maior celeiro de publicações na área, uma pesquisa mais abrangente poderia levar a outros achados; e o trabalho não levou em consideração a qualidade dos artigos avaliados por softwares especializados como "Publish or Perish".

\section{REFERÊNCIAS}

ANDRADE, A.; ROSSETTI, J. P. Governança Corporativa: Fundamentos, Desenvolvimento e Tendências. 6. ed. São Paulo: Atlas, 2012.

BRITO, O. Controladoria de risco - retorno em instituições financeiras. São Paulo: Saraiva, 2003.

CERVO, A.L; BERVIAN. P.A; SILVA, R. Metodologia Científica. 6a ed. São Paulo: Pearson. 2007.

CHEN, Y. CHONG, P.P, TONG, M.Y. The Simon-Yule approach to bibliometric modeling. Information Processing \& Management, v. 30, n. 4, p. 535-56. 1994. Disponível em: http://www.sciencedirect.com/science/article/pii/0306457394900388. Acessado em: 12 de dez. de 2015.

COCURULlO, A. Gestão de Riscos Corporativos: Riscos Alinhados com algumas ferramentas de gestão - Um estudo de caso. 3. ed. São Paulo: Universidade São Francisco (USF), 2004.

COSO-Committee of Sponsoring Organizations of the Treadway Commission. Gerenciamento de Riscos Corporativos - Estrutura Integrada, 2007. Disponível em: http://www.coso.org/documents/COSO ERM ExecutiveSummary Portuguese.pdf. Acesso em: 09 set. 2012.

GITMAN, L. J. Princípios de Administração Financeira. 12. ed. São Paulo: Pearson Prentice Hall, 2010.

GODOY, A. S. Introdução à pesquisa qualitativa e suas possibilidades. Revista de Administração de Empresas, v. 35, n. 2, 57-63, 1995. Disponível em http://www.scielo.br/pdf/rae/v35n2/a08v35n2.pdf. Acesso em: 28 mai. 2015.

HALL, R. H. Organizações: Estruturas, processos e resultados. 8. ed. São Paulo: Pearson Prentice Hall, 2004.

HANDZIC, M. Integrated socio-technical knowledge management model: an empirical evaluation". Journal of Knowledge Management, Vol. 15 Iss 2 pp. 198 - 211, 2011. Disponível em: < http://www.emeraldinsight.com/doi/pdfplus/10.1108/13673271111119655>. Acesso em: 28 mai. 2015.

JORDAN, S.; JORGENSEN, L; MITTERHOFER, H. Performing risk and the project: Risk maps as mediating instruments. Management Accounting Research, v. 24, 2013 pp 156-174. Disponível em: www.elsevier.com/locate/mar. Acesso em: 01 mai. 2014. 
LIRA, A. M.; PARISI, C.; PELEIAS, I. R.; PETERS, M. R. S. Uses of ERP Systems and their influence on Controllership functions in Brazilian Companies. JISTEM - Journal of Information Systems and Technology Management. Revista de Gestão da Tecnologia e Sistemas de Informação Vol. 9, No. 2, May-Aug. 2012, pp.323-352. ISSN online: 1807-1775. Universidade de São Paulo. Disponível em http://www.jistem.fea.usp.br/index.php/jistem. Acesso em: 27 abr. 2014.

MORGAN, G. Imagens da organização. São Paulo: Atlas, 1996.

MOSIMANN, C. P; FISCH, S. Controladoria: Seu papel na administração de empresas. 2. ed. São Paulo: Atlas, 2008.

MOTTA, F. C. P.; VASCONCELOS, I. F. G. Teoria Geral da Administração. São Paulo: Pioneira Thomson Learning, 2002.

NASCIMENTO, A.M; OTT, ERNANI; SILVA. L.M; Sistemas de Controles Internos. In: NASCIMENTO, A. M., REGINATO L. (Organizadores). Controladoria: um enfoque na eficácia organizacional. São Paulo: Ed. Atlas, 2007.

PADOVEZE, C. L. Contabilidade Gerencial: Um enfoque em sistema de informação contábil. 7ạ ed. São Paulo: Atlas, 2010.

PADOVEZE, C. L. Controladoria Estratégica e Operacional. 3a. Ed. São Paulo: Cengage Learning, 2012.

PARKER, B. Evolução e Revolução: Da Internacionalização a Globalização. In: CLEGG, S. R.; HARDY, C.; NORD, W. R. Handbook de Estudos Organizacionais: modelos de análise e novas questões em estudos organizacionais. Organizadores da edição brasileira: CALDAS, M., FACHIN, R., FISCHER, T. v. 1, Cap. 15, p. 00-4433. São Paulo: Atlas, 1999.

PARSONS, T. Sociedades: Perspectivas Evolutivas e Comparativas. São Paulo: Prentice Hall, 1966.

PARSONS, T. O Sistema das Sociedades Modernas. São Paulo: Prentice Hall, 1974.

PEREIRA, C. A. Ambiente, Empresa, Gestão e Eficácia. In: CATELLI, A. (Coordenador). Controladoria: uma abordagem da gestão econômica - GECON. 2. ed. São Paulo: Atlas, 2001.

PETERS, H. P. F., \& VAN RAAN, A. F. J. (1993). Co-word-based science maps of chemical engineering. Part I: Representations by direct multidimensional scaling. Research Policy, 22(1), 23-45. Disponível em: http://www.sciencedirect.com/science/article/pii/ $004873339390031 \mathrm{C}$. Acessado em 20 maio de 2016.

PONDY, L. R; CONOLLY, T. Systems, Knowledge and Organizational Development. Journal of Organizational Change Management. v. 2, n. 2, p. 30-39, 1989. Disponível em: http://www.emeraldinsight.com/doi/pdfplus/10.1108/EUM0000000001180. Acesso em: 28 mai. 2015.

PRADO, E.V. Práticas de gerenciamento de riscos corporativos: um estudo de caso em uma indústria multinacional de autopeças. Dissertação (Mestrado em Administração) - Faculdade de Gestão e Negócios / Programa de Pós-Graduação em Administração - Universidade Metodista de Piracicaba, 2014.

RESCH, S.; FARINA, M. C. Mapa do Conhecimento em Nanotecnologia no Setor Agroalimentar. In SIMPÓSIO DE GESTÃO DA INOVAÇÃO TECNOLÓGICA, 28., 2014, Belo Horizonte. Anais... Rio de Janeiro: ANPAD, 2014. 
RIP, A.; COURTIAL, J.P. Co-word maps of biotechnology: An example of cognitive scientometrics. Scientometrics, v. 6, n. 6, p. 381-400, 1984. Disponível em: http://doc.utwente.nl/57102/. Acessado em 25 abril de 2016.

SANTOS, N.C., FRANCISCHETTI, C.E., SACOMANO NETO, M., PADOVEZE, C., SPERS, V. R. E. Modelo Econômico-Contábil-Financeiro para diagnóstico organizacional. Revista de Administração da UNIMEP. v.11, n.2, Maio/Agosto - 2013. Disponível em http://www.raunimep.com.br/ojs/index.php/regen/article/view/649/536. Acesso em: 05 jul. 2015.

SHAH, S. K.; CORLEY, K. G. Building better theory by bridging the quantitative-qualitative divide. Journal of Management Sudies, v. 43, n. 8, p. 1821-1835, 2006. Disponível em http://onlinelibrary.wiley.com/doi/10.1111/i.1467-6486.2006.00662.x/pdf. Acesso em 28 mai. 2015.

VAN ECK, N. J.; WALTMAN, L. (2007). VOS: A new method for visualizing similarities between objects. In H.-J. Lenz \& R. Decker (Eds.), Advances in data analysis: Proceedings of the 30th annual conference of the German Classification Society (pp. 299-306). Heidelberg: Spring

VANTI, N. A. P. Da bibliometria à webometria: uma exploração conceitual dos mecanismos utilizados para medir o registro da informação e a difusão do conhecimento. 2002. Disponivel em: http://www.scielo.br/pdf/ci/v31n2/12918.pdf. Acessado em 13 de dezembro de 2015.

WESTON, F. J.; BRIGHAM, E. F.; Fundamentos da Administração Financeira. 10. ed. São Paulo: Pearson Makron Books, 2004.

ZITT, M.; BASSECOULARD, E.; OKUBO, Y. Shadows of the past in international cooperation: Collaboration profiles of the top five producers of science. Scientometrics, v. 47, n. 3, p. 627657. 2000. Disponível em: http://www.akademiai.com/doi/abs/10.1023/ A\%3A1005632319799?journalCode=11192. Acesso em: 10 maio de 2016. 